\title{
EMPATHIC CARE: NEW PERSPECTIVES
}

\author{
Nicoleta Răban-Motounu ${ }^{1, *}$ \\ ${ }^{1}$ University of Pitești, Department of Psychology, Communication Sciences, and Social Assistance, \\ Târgu din Vale, No.1, Pitești, Argeș, Romania
}

\begin{abstract}
Empathy studies have revealed new aspects of the concept with various implications in health psychology, psychopathology, education, developmental psychology, and medical care. The study of the biological correlates of empathic relating tend to highlight the effects in several fields, from close interpersonal relationships to professional ones. The Diagnostic and Statistical Manual of Mental Disorders, the Vth edition, uses empathy as a significant dimension to characterize the personality disorders, which are no longer separated from the other previously categorized as Axis I disorders. The objective of the present paper was to come with a literature review focused on the new directions opened in health psychology and developmental psychology as suggested by the discoveries from empathy studies, followed by a synthesis intended to help its understanding and to come up with interventions aiming at empathy development. Previous studies, starting from supporting development in the first years of life, continuing with the analgesic effects of empathic relating, or on the activity of the immune system will be discussed. An analysis in the context of mental disorders may help identify the dimensions to be targeted in interventions, so that the person may harmoniously interact with others. In the context of somatic disorders, empathy helps in understanding the person's suffering, especially when she does not have the means to express it adequately. Moreover, empathic care may be used for pain relief in close interpersonal relationships, helping in mobilizing the organism 's resource to face a challenge.
\end{abstract}

Keywords: compassion, emotional awareness, emotional contagion, pain

\section{EMPATHY: SELF AND SELF-IN RELATIONSHIP}

Empathic relating and compassion play an essential role in child care, but also at a societal level, as a basis for prosocial behavior and support for the disadvantaged or the suffering. In the case of mental disorders, the capacity for empathy, with its several components (emotional empathy, cognitive empathy, perspective taking, the theory of mind, personal distress, and compassionate behavior) is taken into consideration as a characteristic of the illness. In psychosomatic disorders, it is considered that psychological problems lead to dysfunctions in the organism, by the experience of certain psychological issues, but promising data also come from the field of medical care for persons with somatic disorders. The objective of the present paper was to come with a literature review focused on the new directions opened in health and developmental psychology as suggested by the discoveries from empathy studies, followed by a synthesis intended to help its understanding and come up with interventions aiming at developing empathy in several contexts.

\section{EMPATHIC CARE FOR PERSONS WITH SOMATIC DISORDERS}

The doctor' empathic interaction with the patient has an impact on the duration of common cold: For those patients who gave perfect scores to the practitioner, cold severity and duration were significantly lower and the increase in the IL-8, an inflammatory cytokine - marker of immune response, at 48 hours, 
was significantly larger (Rakel, Hoeft, Barrett, Chewning, Craig, \& Niu, 2009). The effects of the empathic practitioner care in common cold appeared not only in face-to-face interactions, but also when the patient received a consultation via telephone: The most important aspect was the patient's feeling of being understood and cared for, when considering also IL-8, and the duration of cold (Rakel, Barrett, Zhang, Hoeft, Chewning, Marchand, Scheder, 2011).

For 2898 patients with moderate to severe pain referred to pain clinics in Spain, the pain relief and the health-related quality of life were predicted by patients' perception of physician empathy and optimism (Canovas et al., 2017). While the pain intensity was moderate to severe at initial assessment and significantly decreased, the patients' levels of perceived empathy indicated moderate to high levels, with no significant changes. The changes in the coping strategies were not statistically significant. Selfassessments of physician's empathy indicated high levels at the end of the study, although they were inversely correlated with changes in pain.

According to a study by Jensen, Gollub, Kong, Lamm, Kaptchuk, and Petrovic (2020) patient's ratings on doctor's interpersonal empathy during consultation had no association with the doctor's personal distress, nor with the activation of brain regions corresponding to the aversive component of experiencing empathy. The relation was robust to the doctor's self-assessment of trait empathic concern, or to the activation of reward related and compassion related brain regions during treatment. This discovery led to considering compassion and pleasure as key factors for successful doctor-patient interactions, especially when dealing with patients' pain.

Tei and colleagues (2014) examined compassion fatigue (or empathy induced burnout) in association with the empathy-related brain activity in medical staff. They found out that reduced empathy-related brain activity explained burnout severity and it was correlated with alexithymia (difficulty in recognizing emotions) and emotional dissonance. Thus, they showed that burnout may be explained by lower emotional awareness and expression.

\section{EMPATHIC CARE IN THE DEVELOPMENT AND CARE FOR PERSONS WITH MENTAL DISORDERS}

Individuals with high-functioning autism show emotional empathy and contagion for pain, but they also have significant increased activity in the areas involved in cognitive reappraisal, involving discrimination between self- and other-related states in perspective taking (Hadjikhani, Zürcher, Rogier et al., 2014). Schore (2014) found that children diagnosed with autism have experienced intersubjective deficits in the mother-infant dyad. A harmonious interaction involves answering with attention focused towards the child starting with his first attempts of social engagement, by visual-sensory and tactilegestural non-verbal communication, followed by auditory-prosodic synchronization with the purpose of self-regulation and interconnection. The mother first empathizes with the child and finds out how he feels, and then prologues the state if it is a positive one or helps in regulating it if it is a negative one. This, after the initial stage, when she empathetically understands his needs and satisfies them, supporting growth and maturation, but also brain development, all accompanied by a positive affective state. This type of interactions may prepare the child for verbal communication in the following stages. Galbusera, Finn, and Fuchs (2018) found that negative symptoms in schizophrenia have a negative correlation with the nonverbal interactional synchrony in the psychotherapeutic relationship and that a 10-weeks body-orientated psychotherapeutic program, centered on increasing this type of synchrony, has effects both on client-psychotherapist synchrony, but also on the negative symptoms (with medium size effects). They explained the results using a theory focused on the subjectivity and intersubjectivity, and their disturbances in persons diagnosed with schizophrenia. 


\section{EMPATHIC CARE IN CLOSE INTERPERSONAL RELATIONSHIPS - THE EXPERIENCE OF PAIN OR POSITIVE EMOTIONS}

Goldstein, Weissman-Fogel, Dumas, and Shamay-Tsoory (2018) organized an experiment to further explore the analgesia related to social touch. They tested the brain-to-brain coupling and synchrony between romantic partners when one of them experiences pain, resulting in pain alleviation in two different conditions: when holding hands or no touch between them and only observing. They found that the observer's empathic accuracy correlates with analgesia magnitude, as the brain-to-brain coupling had increased (the central regions for the person experiencing pain and the right hemisphere of the pain observer). It is important to mention also that the brain-to-brain coupling happened only in the alpha band. One of their explanations was that touch extended the areas activated by pain and empathic reactions, thus, favoring reciprocal understanding. When none of the partners experienced pain, nor they touched, the interbrain coupling was weaker, the weakest effect being experienced when a partner experienced pain but they did not touch each other, with the analgesia and the partner's empathic accuracy also being the lowest. Previously, Coan, Schaefer, and Davidson (2006) showed that the effects of spousal hand-holding on pain experience varies as a function of marital quality, and the brain effects did not appear when holding the hand of a stranger. It seems that a resonance effect appears, the analgesia being experienced when the partner holding hand succeeds in remaining calm, as she or he accurately understands the intensity of the pain, and the suffering one also experiences calmness.

The cognitive load reduces caregiving intention and increases intention to perpetrate child neglect, with state empathy interacting with the cognitive load to predict the same variables (Hiraoka and Nomura, 2016). The cognitive load was manipulated by introducing a memory task consisting in remembering meaningless English alphabet strings, that the participants were instructed to memorize while hearing infant crying. Personal distress was positively associated with intention to perpetrate neglect and negatively associated with the intention to provide care. This may suggest that the meaningless cognitive task may interfere with the cognitive aspects of empathy, like perspective taking and correctly using the theory of mind, while the awareness field is occupied by the information in the task. In a previous study, Lin and McFatter (2012) showed that personal distress and empathy were positively related to the intention to intervene. It is important to note, though, that, in the Hiraoka and Nomura's experiment, the participants only heard the infant crying, without actually seeing him, while Lin and McFatter's research design involved a 1-minute-long video of infant crying, actually seeing an infant experiencing such an intense distress that it makes him cry. But we may add that the participants in both experiments rated their cognitive appraisal of the intent to provide care, while they did not have any control over the child's state (the distress ceased with the end of the experimental task). The discrepancy between a person's actual behavior in a situation and how she thought she should have behaved is negatively associated with the personal well-being and positively with negative affectivity (Chrystal, Karl, Fischer, 2019). This is in line with the previous study regarding the physician's self-assessed traitempathic concern, which correlated with the patients' assessment of empathic care, but also with the practitioner's assessment of empathic care.

Tomova, Majdandzic, Hummer, Windischberger, and Lamm (2017) discovered that stress increases the tendency to share other people's pain automatically (contagion), but it also increases inappropriate other-related responses when the situation requires taking the perspective of others and regulation of automatic affective responses. We may simply understand that the person accumulates internal pain from others automatically, with less ability to self-regulate or to help others.

Another study involved watching photos of people experiencing sadness, happiness, and anxiety in four experimental conditions (just watching them; responding to them as if they would have seen the photo in a magazine, at home; imagining how the person in the photo might feel in the situation and how it would affect her life; and a memory task added to that). The cognitive loading had a negative impact on 
the activation in regions related to social cognition and empathy (Morelli \& Lieberman, 2013). Also, administering painkillers reduces empathy to other people's positive experiences (Mischkowski, Crocker, \& Way, 2019), but also for pain (Mischkowski, Crocker, \& Way, 2016).

\section{SOMATIC BASIS OF EMPATHY AND SOCIAL RELATIONSHIPS}

Empathy research showed that it is a more complex phenomenon than expected. The mirror neurons allow the intuitive, automatic resonance with another in the visual field, the anticipation of the next move and the understanding of the other's intention. More complex cognitive mechanisms are involved in adopting and understanding the other's perspective. Interpersonal resonance also allows the understanding and the regulation of the child's affective state by the mother, thus developing internal mechanism for affective self-regulation, involving deep, physiological reactions (Shore, 2014).

Alcalá-López and colleagues (2017) characterized the social brain as containing 36 areas organized on four hierarchical levels: the visual-sensory cluster, the limbic cluster, an intermediate level cluster, and a higher-level cluster. One important specification is that, while the lower levels have connections with higher levels in general, the areas forming the higher-level cluster are mostly connected among each other than with functional seeds from intermediate or lower-level clusters. The connections among mirror neurons and empathy-related seeds "were prominent up to the point of forming the shared intermediate-level cluster" (p.2226). Mirror neurons have the same spiking activity as the person observed, when passively observing her acting towards the object satisfying her need. This enables the observer to understand her intentions and to act just the same, with the condition that both the person observed and the object of her need being in the visual field at the same time. Their results confirm that empathy involving these intermediate level areas has more to do with embodied simulation than with the theory of mind, with its abstract emulation. Their model shows that social cognition is an ongoing process, more active during a task than in a resting state, involving integration of somatic-sensory and associative processing. The higher-level cluster of connectionally-coherent seeds, mostly associated with the theory of mind (the knowledge about how the human mind works), constantly receive information about the ongoing social events, updating internal models of social-phenomena. AlcaláLópez, Vegeley, Binkofski, and Bzdok (2019) showed that persons with a diagnosis of schizophrenia are sensible to the emotional experience of another by intuitive empathy, characterized by the activation of mirror neurons, but they have difficulties in taking the perspective of another, mainly in understanding intersubjectivity. Higher scores in personal distress, but also significantly lower scores on perspective taking have been encountered also in persons with bipolar disorder (Cusi, Maqueen, \& McKinnon, 2010).

\section{CONCLUSIONS}

Practitioner's empathic understanding of patient's suffering and his care for his well-being, results in rapid and modulated immune reactions, in common cold especially, which reduces suffering and illness duration. It is correlated more with the practitioner's self-assessment of trait empathic concern in general and not with the practitioner's distress. The cognitive dissonance between the internal values and the actual empathic behavior may result in negative affect for the practitioner. As for physicians experiencing burnout, it is associated with less activity in the empathy related areas. In close interpersonal relationships the pain is eased by synchronization of cerebral activity when the other both accurately estimates the pain and succeeds in remaining calm (suggesting that he correctly understands the pain as being experienced by the significant other), a process similar with self-regulation of negative affect in infancy. Increased stress, consisting in irrelevant cognitive load when seeing somebody suffering, increases personal distress and inappropriate responses, and leads to neglect. Administration of painkillers also affects the empathic response. There is a higher level of interconnected brain areas, 


\section{Current Trends in Natural Sciences}

Vol. 9, Issue 18, pp. 189-193, 2020

https://doi.org/10.47068/ctns.2020.v9i18.025

Current Trends in Natural Sciences (on-line)

ISSN: 2284-953X

Current Trends in Natural Sciences (CD-Rom)

ISSN: 2284-9521

ISSN-L: 2284-9521

ISSN-L: 2284-9521

superior to those used in empathic processing, more connected with the theory of mind, suggesting the accumulation and refinement, in a process of meaning making at an abstract level, of personal social experience, which may refer more to the own functioning, involved in proper understanding of self and other's state in interaction. Self-awareness and awareness techniques, especially being aware of own's empathic capacity and emotions, involved in self-support and interpersonal relationships, may support the process of empathic relating for parents, psychotherapists, and medical staff, resulting in mutual positive affect.

\section{REFERENCES}

Alcalá-López, D., Vegeley, K., Binkofski, F., \& Bzdok, D. (2019). Building Blocks of Social Cognition: Mirror, Mentalize, Share. Cortex, 118, 4-18. doi: 10.1016/j.cortex.2018.05.006

Alcalá-López. D, Jeffries, E., Smallwood, J., Van Overvalle, F., Vogeley, K., Mars, R. et al. (2017). Computing the Social Brain Connectome Across Systems and States. Cerebral Cortex, 28(7), 2207-2232. Doi:10.1093/cercor/bhx121

Coan, J.A., Schaefer, H.S., \& Davidson, R.J. (2006). Lending a Hand: Social Regulation of the Neural Response to Threat. 17, 1032-1039. doi:10.1111/j.1467-9280.2006.01832.x

Canovas, L., Carrascosa, A.J., Garcia, M., Fernandez, M., Calvo, A., Monsalve, V., Soriano, J.F. (2017). Impact of Empahty in the Patient - Doctor Relationship on Chronic Pain Relief and Quality of Life: A Prospective Study in Spanish Pain Clinics. Pain Medicine, 19(7), 1304-1314. doi:10.1093/pm/pnx160

Chrystal, M., Karl, J.A., Fischer, R. (2019). The Complexities of "Minding the Gap": Perceived Discrepancies Between Values and Behavior Affect Well-Being. Forntiers in Psychology, 10.736. Doi:10.3389/fpsyg.2019.00736

Cusi, A.M., MacQueen, G.M., \& McKinnon, M.C. (2010). Altered self-report of empathic responding in patients with bipolar disorder. Psychiatry Research, 178(2), 354-358. doi: 10.1016/j. Psychres.2009.07.009

Galbusera, L., Finn, M.T., \& Fuchs, T. (2018). Interactional synchrony and negative symptoms: An outcome study of bodyoriented psychotherapy for schizophrenia. Psychotherapy Research. 28(3), 457-469. Doi: 10.1080/10503307.2016.1216624

Goldstein, P., Weissman-Fogel, I., Dumas, G., \& Shamay-Tsoory, S.G. (2018). Brain-to brain coupling during handholding in associated with pain reduction. Proceedings of the National Academy of Sciences of the United States of America, 115(11), E2528-E2537. doi:10.1073/pnas.1703643115

Hadjikhani, N., Zürcher, N.R., Rogier, O., Hippolyte, L., Lemonnier, E. et al. (2014). Emotional contagion for pain is intact in autism spectrum disorders. Translational Psychiatry, 4, e343. doi:10.1038/tp.2013.113

Hiraoka, D, Nomura, M. (2016). The Influence of Cognitive Load on Empathy and Intention in Response to Infant Crying. Scientific Reports, 6, 28247. doi:10.1038/srep28247

Jensen, K., Gollub, R.L., Kong, Lamm, C., Kaptchuk, T.J., Petrovic, P. (2020). Reward and empathy in the treating clinician then the neural correlates of successful doctor-patient interactions. Translational Psychiatry, 10, 17. doi:10.1038/s 41398- 020- 0712- 2

Lin, H.C., McFatter, R. (2012). Empathy and distress: Two distinct but related emotions in response to infant crying. Human Behavior and Development, 35(4), 887-897. doi:10.1016/j.infbeh.2012.08.001

Mischkowski, D., Crocker, J., \& Way, B.M. (2016). From painkiller to empathy killer: acetaminophen (paracetamol) reduces empathy for pain. Soc Cogn Affective Neuroscience, 11(9), 1345-1353. doi:10.1093/scan/nsw057

Mischkowski, D., Crocker, J., \& Way, B.M. (2019). A social Analgesic? Acetaminophen (Paracetamol) Reduces Positive Empathy. Frontiers in Psychology, 10, 538. doi:10.3389/fpsyg.2019.00538

Morelli, S.A., Lieberman, M.D. (2013). The role of automaticity and attention in neural processes underlying empathy for happiness, sadness, and anxiety. Frontiers in Human Neurosciences 7, 160, doi:10.3389/fnhum.2013.00160

Rakel, D.P., Hoeft, T.J., Barrett, B.P., Chewning, B.A., Craig, B.M., Niu, M (2009). Practitioner Empathy and the Duration of Common Cold. Family Medicine, 41(7), 494-501

Rakel, D.P., Barrett, B.P., Zhang, Z., Hoeft, T., Chewning, B., Marchand, L., Scheder, J. (2011). Perception of Empathy in the Therapeutic Encounter: Effects on the Common Cold. Patient Education and Counselling, 85(3), 390-397. doi: 10.1016/j.pec.2011.01.009

Schore, A.N. (2014). Early interpersonal neurobiological assessment of attachment and autistic spectrum disorders. Frontiers in Psychology, 5, 1049. doi:10.3389fpsyg.2014.01049

Tei, S., Becker, C., Kawanda, R., Fujino, J., Jankowski, K.F., Sugihara et al. (2014). Can we predict burnout severity from empathy-related brain activity? Translational Psychiatry, 4, e393. doi:10.1083/tp.2014.34

Tomova, L., Majdandzic, J., Hummer, A., Windischberger, C., Lamm, C. (2017). Increased neural responses to empathy for pain might explain how acute stress increases prosociality. Social Cognitive and Affective Neuroscience, 12(3), 401408. doi:10.1093/scan/nsw146

http://www.natsci.upit.ro

*Corresponding author, E-mail address: nicoleta.motounu@upit.ro 